\title{
Mesenteric-Based Surgery Exploits Gastrointestinal, Peritoneal, Mesenteric and Fascial Continuity from Duodenojejunal Flexure to the Anorectal Junction - A Review
}

\author{
J. Calvin Coffey ${ }^{\mathrm{a}, \mathrm{b}}$ Mary Dillon ${ }^{\mathrm{a}}$ Rishabh Sehgal ${ }^{\mathrm{a}}$ Peter Dockery $^{\mathrm{c}}$ \\ Fabio Quondamatteo $^{d}$ Dara Walsh $^{\text {a }}$ Leon Walsh $^{\mathrm{a}}$ \\ ${ }^{a}$ Department of Surgery, University Hospital Limerick, Graduate Entry Medical School, ${ }^{\mathrm{b}} 4 \mathrm{i}$ Centre for Interventions \\ in Infection, Inflammation and Immunity, Graduate Entry Medical School, University of Limerick, Limerick, and \\ 'Anatomy, School of Medicine, NUIG, d'Skin and ECM research group, Anatomy, NUI, Galway, Ireland
}

\section{Key Words}

Complete mesocolic excision $\cdot$ Mesentery $\cdot$ Colon cancer

\begin{abstract}
Introduction: It is now well established that mesentericbased colorectal surgery is associated with superior outcomes. Recent anatomic observations have demonstrated that the mesenteric organ is contiguous from the duodenojejunal to the anorectal junction. This led to similar observations in relation to associated peritoneum and fascia. The aim of this review was to demonstrate the relevance of the contiguity principle to resectional colorectal surgery. Methods: All literature in relation to mesenteric anatomy was reviewed from 1873 to the present, without language restriction. Results: Mesenteric-based surgery (i.e. complete mesocolic excision, total mesocolic and mesorectal excision) requires division of the peritoneal reflection (i.e. peritonoto$\mathrm{my}$ ), and mesenteric mobilisation in the mesofascial plane. These are the fundamental technical elements of mesenterectomy. Mesenteric, peritoneal and fascial contiguity mean that in resectional surgery, these technical elements can be reproducibly applied at all levels from the origin at the superior mesenteric root, to the anorectal junction. Conclusions: The goals of complete mesocolic, total mesocolic and meso-
\end{abstract}

rectal excision can be universally achieved at any level from duodenojejunal flexure to anorectal junction, by adopting technical elements based on mesenteric, peritoneal and fascial contiguity.

(c) 2015 S. Karger AG, Basel

\section{Introduction}

Mesenteric-based surgery includes total mesocolic excision, total mesorectal excision and complete mesocolic excision [1-7]. A substantialbody ofliterature has emerged demonstrating the technical and outcome-related superiority of these approaches $[8,9]$. The goals of mesentericbased surgery are intact and extensive mesenterectomy [5]. The planes utilised are increasingly referred to (based on histologic observations) as the colo- and mesofascial plane $[10,11]$. Recent developments in surgical anatomy enable clarification of the anatomy of the peritoneal, colonic, mesenteric and fascial components of the colo and mesofascial planes [12]. The central tenet is that of ana-

Based on a lecture delivered at the 26th Annual David G Jagelmann/36th Annual Rupert B. Turnbull International Symposium on Colorectal Surgery, Fort Lauderdale, Fla., USA, February, 2015.

\section{KARGER 125}

(c) 2015 S. Karger AG, Base

0253-4886/15/0324-0291\$39.50/0

E-Mail karger@karger.com

www.karger.com/dsu
Prof. J. Calvin Coffey

Department of Surgery, University Hospital Limerick

Graduate Entry Medical School, University of Limerick

Dooradoyle, Raheen, Limerick (Ireland)

E-Mail calvin.coffey@ul.ie 
Fig. 1. a The colofascial plane as seen in a cadaveric dissection. The left colon is visualised. The left lateral peritoneal reflection has been divided to expose the interface (i.e. plane) formed by colon and Toldt's fascia. $\mathbf{b}$ The mesofascial plane as seen in a cadaveric dissection. The left mesocolon is apparent. The left colofascial interface was separated to expose the interface (i.e. plane) formed by mesocolon and underlying left mesocolic fascia.

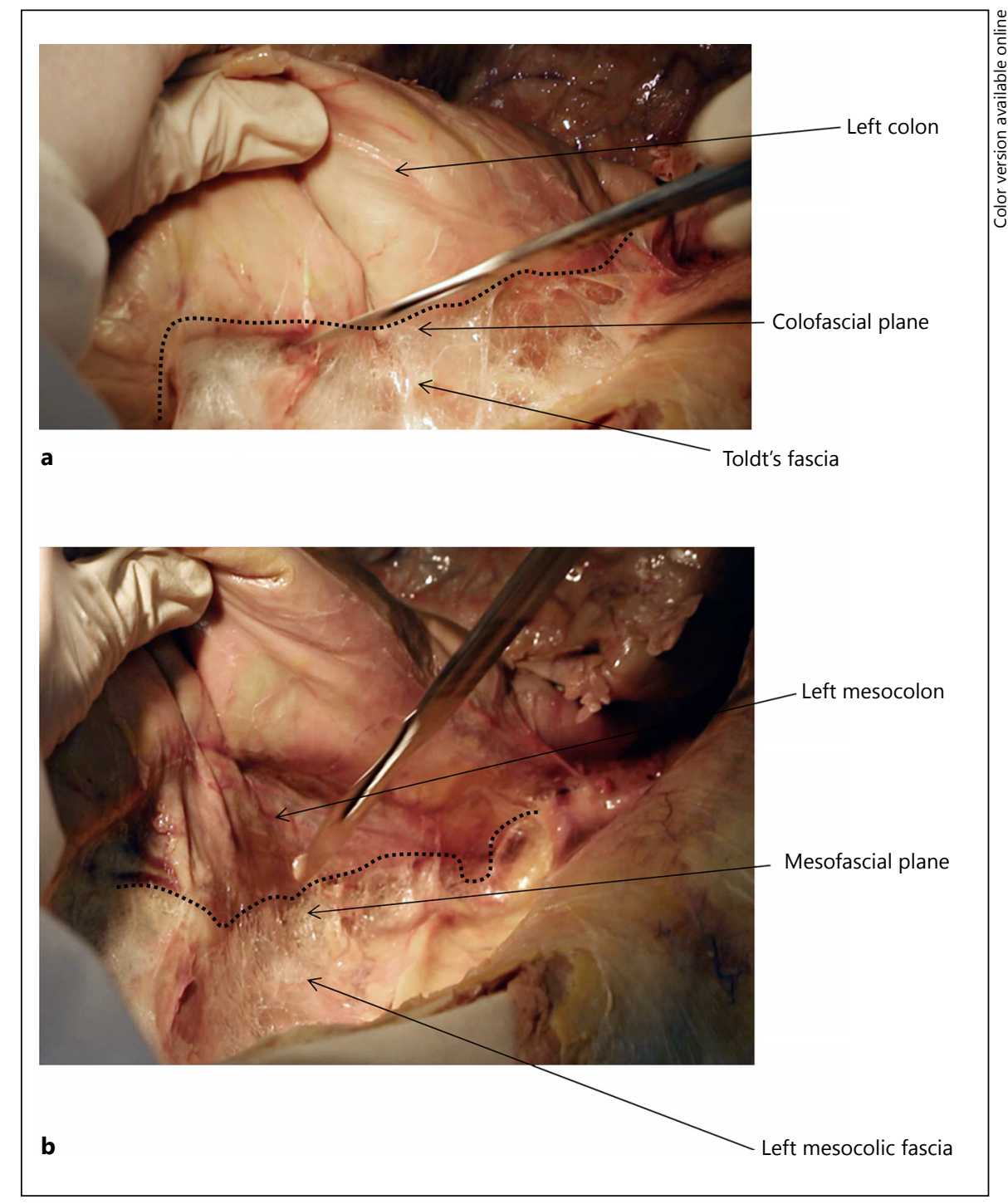

tomic contiguity from the origin of the mesenteric organ to the mesorectum $[10,11]$. This anatomic simplification provides the colorectal surgical community with a universally applicable anatomic basis (even in cases of mal or non-rotation) for resectional colorectal surgery. The following review summarises these observations and their relevance to surgical techniques.

\section{Definition of a Surgical Plane}

Before commencing it is necessary to first define a surgical plane. This has been elegantly described by Professor Bill Heald as the interface between two contiguous surfaces [1]. Importantly, a plane is not formed by a single structure or two structures, but rather by the interface where they are apposed. This is arguably one of the most important definitions in surgery in general, and is the cornerstone of anatomic surgery. As mentioned earlier, mesenteric-based surgery exploits two planes: the colofascial plane is formed by the colon and apposed fascia, while the mesofascial plane is formed by the mesentery and apposed fascia $[1,11,13]$.

\section{The Colo-Fascial Plane}

This is the plane formed by the colon and underlying fascia (fig. 1). A colo-fascial plane occurs wherever the colon is apposed to the posterior abdominal wall (i.e. 
right and left colon and at the flexures). While division of the right lateral peritoneal reflection exposes the plane on the right, division of the left lateral peritoneal reflection exposes the plane on the left. In addition, division of the ileocaecal, splenocolic and hepatocolic peritoneal reflections exposes the colofascial interface in these regions. Once the right lateral peritoneal reflection is divided, this interface is exposed. When the surgeon places it under stretch, by lifting it away from the retroperitoneum, the fascia can either be swept away from the colon, diathermy or sharply divided. By continuing this process, the colon is freed to the point where the mesofascial plane becomes apparent [14]. While this is self-evident, and separation has been practiced for well over one hundred years by gastrointestinal surgeons, the colo-fascial plane has been virtually ignored. It is an important plane to conceptualise, not least because it must be separated for mobilisation, but also because it is the first plane encountered in lateral to medial mobilisation during mesocolic excision.

\section{Mesenteric Component of the Mesofascial Plane}

The classic appraisal of mesenteric anatomy was described by Sir Frederick Treves and indoctrinated dogmatically in anatomic, embryologic and colorectal surgical texts over the past century [15]. The central tenet is that the mesentery is a discontinuous and fragmented structure, and while the transverse mesentery, small bowel mesentery and mesosigmoid are substantive entities, the right and left mesocolon disappear or become vestigial [15]. Our current appraisal, first laid down approximately 2 years ago, describes the mesenteric origin (i.e. root) where the superior mesenteric artery issues from the pancreatic bed. From this location, the mesenteric organ fans out to span the entire gastrointestinal tract from the duodenal-jejunal junction to a termination at the distal mesorectum $[10,13,16]$. Where it (the mesentery) is apposed to the retroperitoneum, its surface area is limited. However, at the gastrointestinal margin, it elongates considerably. Approximately 6 feet of elongated mesentery is compactly plicated into the abdominal cavity and tightly packaged in a spiral confirmation.

Although this description is a refutation of classic and widely held anatomic appraisals, it is not novel. Leonardo da Vinci drew the mesenteric organ as a contiguous structure. To generate this image, da Vinci most likely amputated the gastrointestinal tract at its intersection with the mesentery. A closer inspection of Da Vinci's illustra-

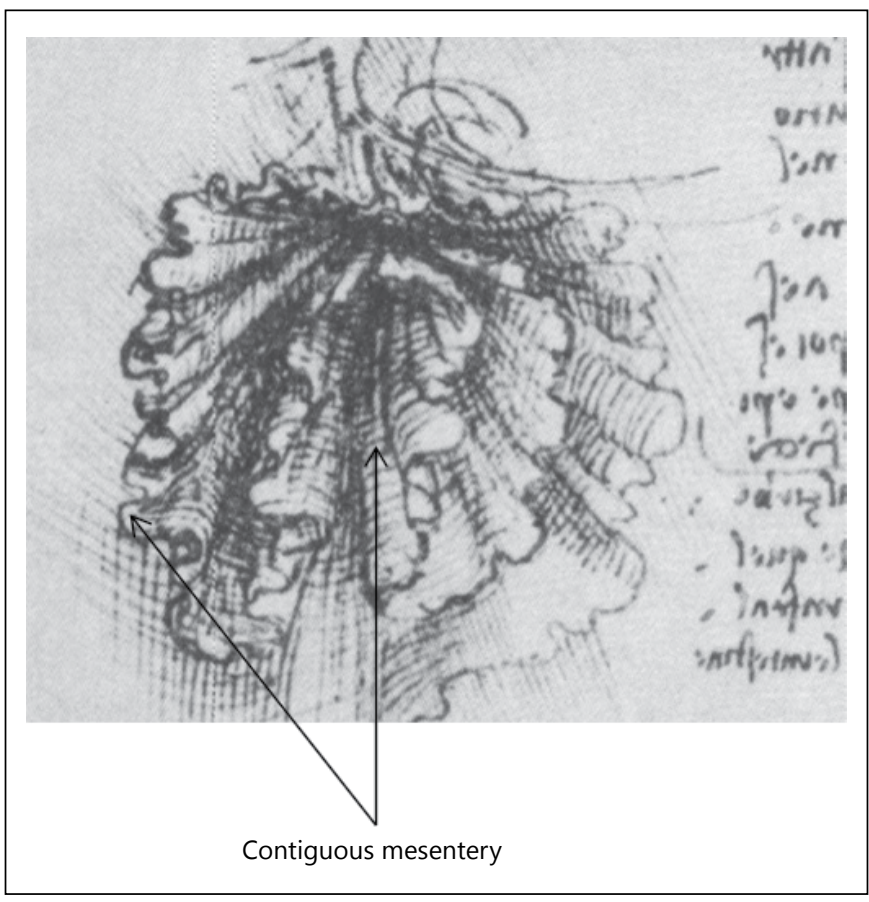

Fig. 2. Drawing of mesenteric organ by Leonardo Da Vinci. It is likely that the gastrointestinal tract was excised at the intersection with the mesentery (i.e. the gastrointestinal margin). Mesenteric contiguity is apparent from the small intestinal mesentery to the mesorectal level.

tion demonstrates a mesenteric convergence towards an unidentified region. We now know that Da Vinci's interpretation was correct, and that the mesentery emerges in a centrifugal manner from a root at the superior mesenteric artery (fig. 2 and $3 a$ ).

One of the best means of demonstrating these anatomical arrangements is cadaveric dissection. Figure $3 \mathrm{a}$ demonstrates the root of the mesenteric organ. Figure $3 \mathrm{~b}$ demonstrates the contiguity between the small intestinal mesentery and the right mesocolon. As can be seen, the small intestinal mesentery and right mesocolon are not discrete entities. Rather they are different regions of the same structure [16]. Similarly, contiguity can be demonstrated between the right and transverse mesocolon at the hepatic flexure and between the transverse and left mesocolon at the splenic flexure (fig. 4) [13, 16]. Finally, the left mesocolon can be demonstrated as it continues distally into the mesosigmoid and thereafter into the mesorectum (fig. 4).

Given mesenteric organ contiguity, there are no boundaries between contiguous regions. This contributes to the confusion that surrounds the structure of this organ. To overcome this, a three-dimensional model was 


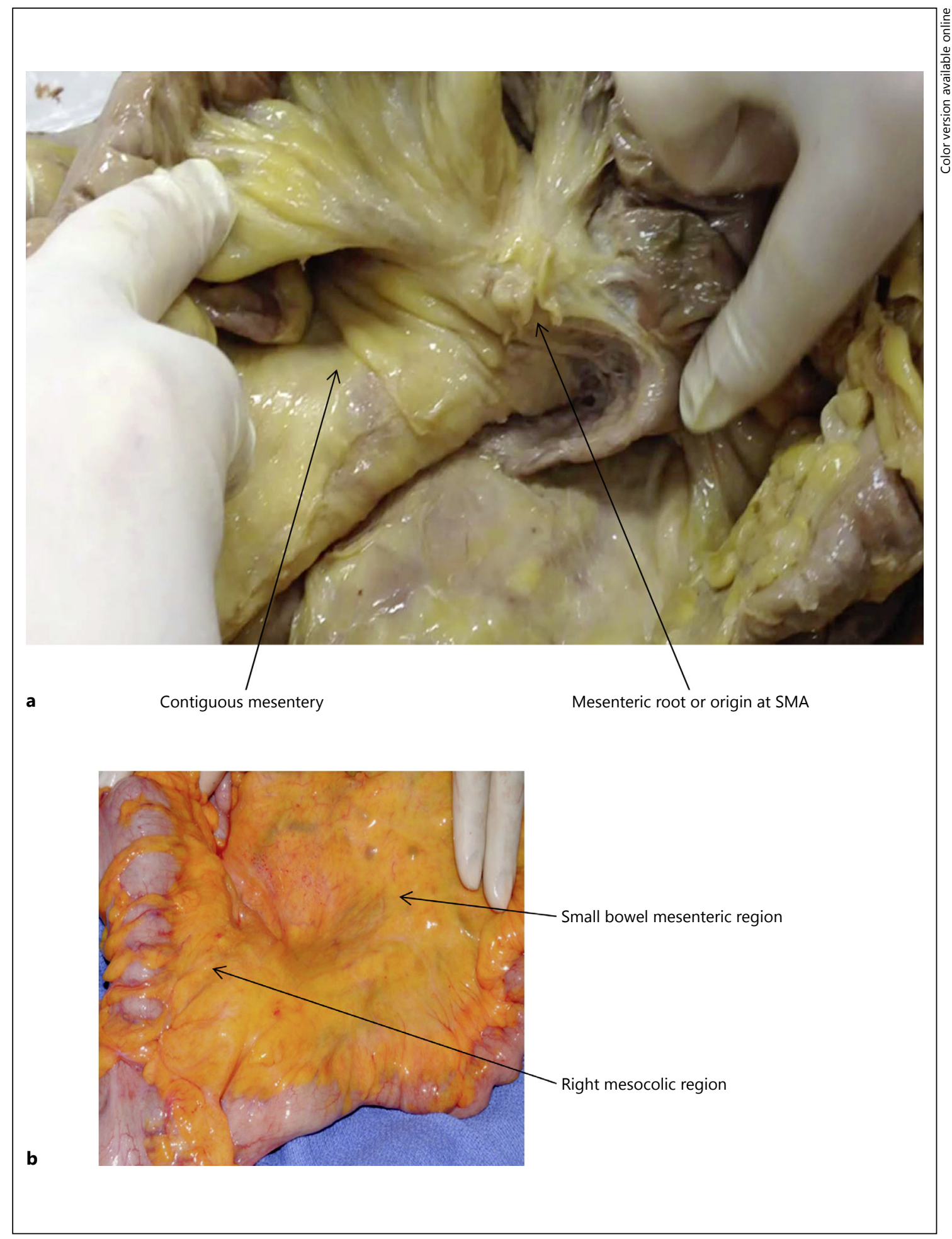

Fig. 3. a Origin (root) of the mesenteric organ in a cadaver in which the mesentery has been fully mobilised. b Intraoperative image demonstrating contiguity between the small intestinal mesentery and right mesocolon. In this case, the mesenteric organ has been mobilised and lifted out of the peritoneal cavity to demonstrate contiguity. 


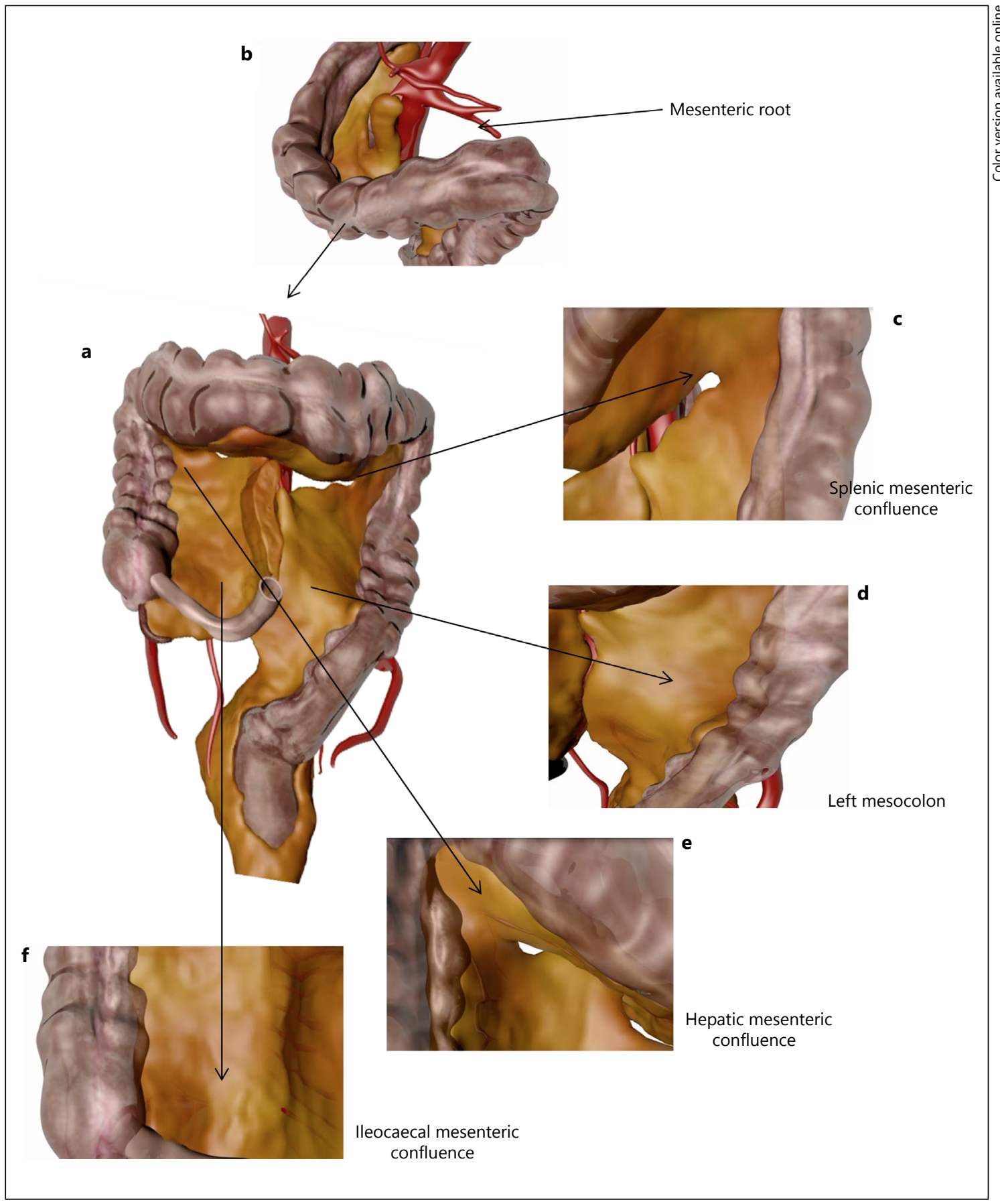

Fig. 4. a 3D digital sculpture of mesenteric organ on left. In each of the surrounding images, the model has been rotated to demonstrate different regions of the mesenteric organ. b View of mesenteric organ from above, demonstrating the root region where the mesenteric organ originates along with the superior mesenteric artery. c The model in a has been rotated and the point of view is directly at the mesenteric component of the splenic flexure. $\mathbf{d}$ The model in a has been rotated such that the point of view is from above, looking down on the left mesocolon. e The model in a has been rotated such that the view provided is directly at the mesenteric component of the hepatic flexure. f The model in a has been rotated such that the view is directly at the junction between the ileum and caecum (the ileocaecal mesenteric confluence). 
digitally sculpted (fig. 4) and rotated to provide direct views of different mesenteric regions. This is an educational modality that is helpful in demonstrating mesenteric contiguity from the root region and around each flexure (fig. 4).

It is only by conducting mesofascial separation (i.e. separating the mesenteric and fascial components intact) that one can demonstrate mesenteric contiguity from origin to termination [12].

\section{Fascial Component of the Mesofascial Plane}

Recently, our group biopsied the right mesocolon in a manner that included the underlying retroperitoneum $[11,17]$. Mesenteric, fascial and retroperitoneal relationships were investigated using a combination of standard histologic staining and scanning electron microscopy [5]. We demonstrated that the mesentery is kept separate from the underlying retroperitoneum by a multi-lamellated fascial layer (i.e. Toldt's fascia). Importantly, only a single fascia occurs in this location. In other words, the concept of separate visceral and somatic fasciae is erroneous. Instead, a single fascial layer is sandwiched between the mesothelium of the under surface of the right mesocolon and mesothelium overlying the retroperitoneum.

Beneath both mesenteric and retroperitoneal mesothelial layers a further submesothelial connective layer occurs. In the case of the mesocolon, the complex of mesothelium and submesothelial connective tissue corresponds to the 'visceral peritoneum'. Some authors use the terms 'visceral fascia' and 'visceral peritoneum' interchangeably [2]. The complex of mesothelium and connective tissue overlying the retroperitoneum has been referred to as the 'parietal peritoneum'. Some authors use the terms 'somatic fascia' and 'parietal peritoneum' interchangeably. While the terms 'visceral' and 'parietal peritoneum' are anatomically appropriate, the terms 'visceral' and 'somatic fascia' are inaccurate as they are not fasciae per se. The surgical plane exploited in complete mesocolic excision (i.e. the mesofascial plane) occurs between a single fascia (i.e. Toldt's fascia) and the overlying visceral peritoneum (i.e. the lower surface of the overlying mesocolon).

Toldt's fascia is variable in appearance. In the mesorectal region, it is areolar in nature. Beneath the mesosigmoid (i.e. mesosigmoidal fascia), it is similarly areolar in appearance. Beneath the left mesocolon (i.e. the left mesocolic fascia), it is more dense due to the presence of multiple lamellae of collagen. As a result, the left mesocolic fascia is more readily separated from the visceral peritoneum of the overlying left mesocolon.

Just as the mesenteric component of the mesofascial interface is contiguous, it is feasible that the fascial component is also contiguous (fig. 5) [16]. This is difficult to demonstrate due to regional variations in composition (see above) and because once the mesocolon has been excised, it bears little structural similarity to its topography in the undisturbed state. The visual human project is a digital archive, which enables the identification of structures in an undisturbed format (fig. 5). Consequently, it permits the identification of fascial planes and associated structures. For example, one can identify the mesorectum and associated mesorectal fascia (fig. 5k). One can then follow this back upwards, out of the pelvis, under the mesosigmoid (mesosigmoidal fascia). Doing this, one observes a contiguity between the mesorectal fascia, the mesosigmoidal fascia (underneath the mesosigmoid), and the left mesocolic fascia (beneath the left mesocolon) (fig. 5, middle and right columns). Thus, these represent different regions of the same fascial entity. In keeping with this, the mesorectal fascia, Gerota's fascia and the anterior pararenal fascia are different regions of the same fascial entity.

\section{Peritoneal Reflections}

As demonstrated earlier, the mesenteric and fascial components of the mesofascial plane are contiguous and substantive entities. Upon entry into the abdomen, they are not immediately apparent. To demonstrate these, the overlying peritoneal reflection must be divided.

In general, in colorectal literature, the peritoneal reflections are identified by the White line of Toldt, and it is recommended that one begins mobilisation or dissection approximately $5 \mathrm{~mm}$ medial to the white line. There are a number of problems associated with this approach. In the first instance, the white line of Toldt is not always present. When it is, it is similar to congenital adhesions that frequently occur between mesenteric and abdominal wall peritoneum.

Our recent observations have demonstrated that wherever the mesenteric organ is apposed to the retroperitoneum (or separates from the retroperitoneum), a peritoneal reflection occurs (fig. 6). In keeping with this, a peritoneal reflection occurs at the base of the small bowel mesentery where it adheres to the posterior abdominal wall and continues laterally as the right meso- 


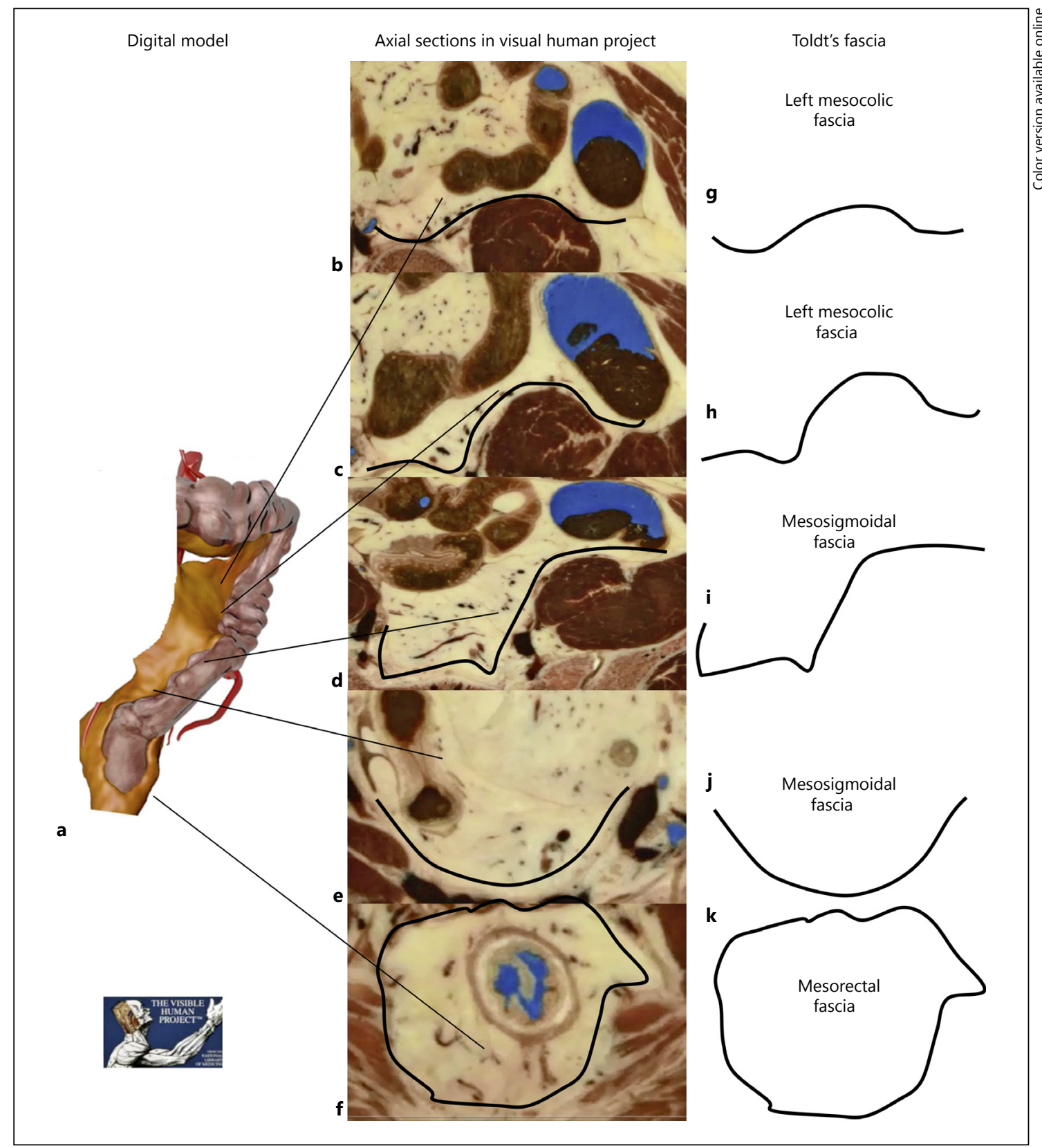

Fig. 5. a 3D digital sculpture showing mesentery and colon from the splenic flexure to the rectum. This serves as a reference for the middle and right-sided column. The middle column (b-f) comprises axial slices derived from the visual human project (VHP) and at the levels indicated in the digital model. A trace is superim- posed on each axial image, which is parallel to Toldt's fascia. In each image, the trace is parallel to but slightly removed from the fascia, in order to illustrate the latter. The right column ( $\mathbf{g - k})$ consists of the individual traces in the middle column (with VHP images removed). colon. To gain access to the mesofascial plane, a peritonotomy of the peritoneal reflection in this location is required. Once this is lengthened, and the interface placed on stretch, it is possible to sharply separate the components of the mesofascial interface without disrupting the integrity of either. These steps (i.e. peritonotomy and mesofascial separation) are key steps in mesentericbased surgery (CME, TMCE and TME).

The peritoneal reflection at the base of the small bowel mesentery continues around the ileocaecal junction 


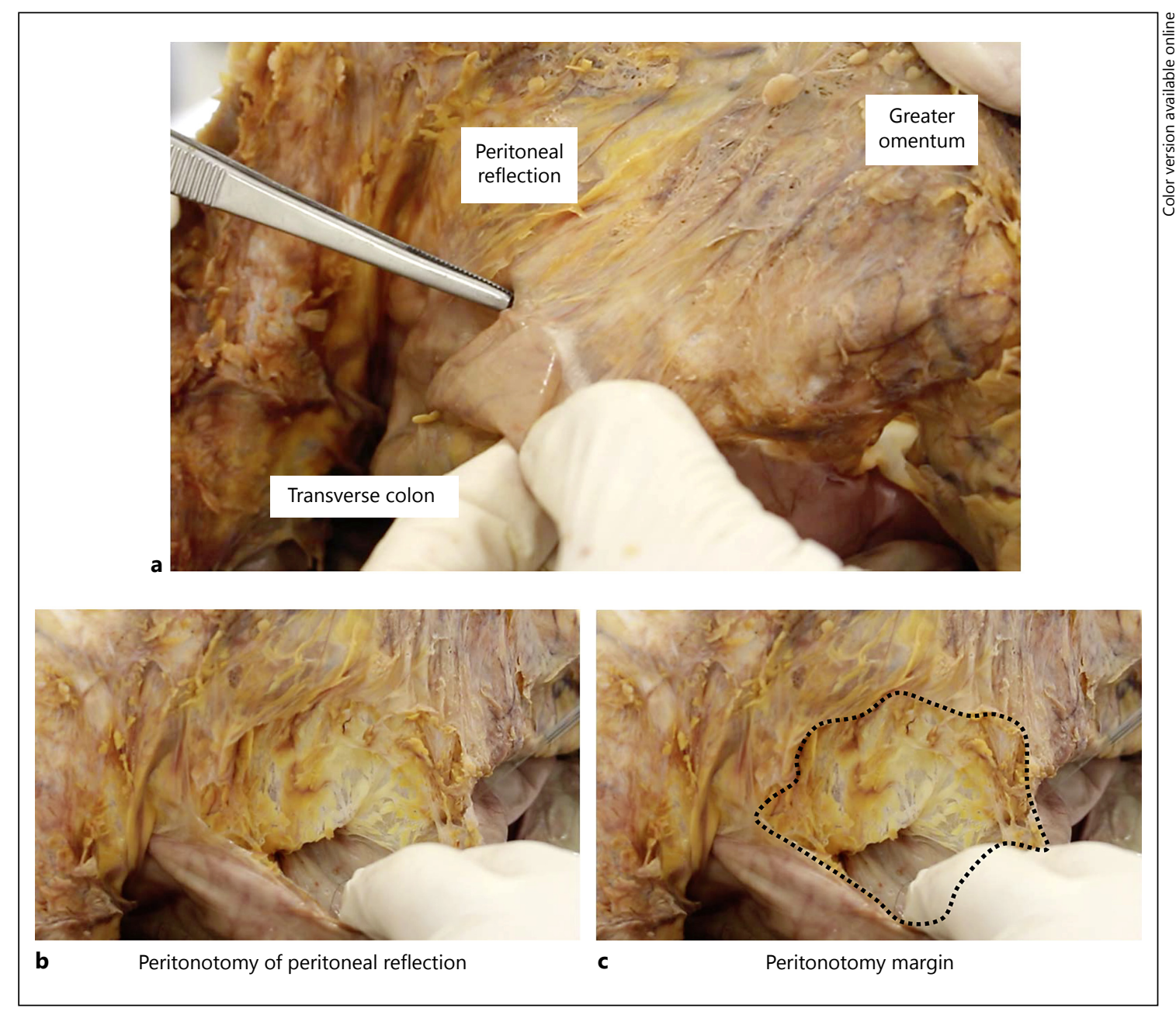

Fig. 6. a Peritoneal reflection between the greater omentum and underlying transverse colon. b, c The peritoneal reflection adjoining omentum and colon has been divided (b) and the free margin of the peritonotomy is outlined (c).

and thereafter along the lateral aspect of the right colon (i.e. the right lateral peritoneal reflection). The right lateral peritoneal reflection continues around the hepatic flexure as the hepatic-colic peritoneal reflection. On the right side, division of the right lateral peritoneal reflection exposes the colo-fascial plane. Separation of colic and fascial components brings the surgeon to the mesofascial plane. Similarly, peritonotomy of the hepatic-colic peritoneal reflection exposes the colo and mesofascial plane at the hepatic flexure and is an early step in hepatic flexure mobilisation.

An unnamed peritoneal reflection also occurs between the greater omentum and underlying transverse colon (fig. 6). This is appreciated intraoperatively by placing the greater omentum under traction and the transverse colon under counter-traction. Division of this reflection exposes the interface formed by the greater omentum and underlying transverse mesocolon (fig. 6). Just as on the right, the splenocolic peritoneal reflection is really a peritoneal reflection that continues distally as the left lateral peritoneal reflection. The left lateral peritoneal reflection continues as the reflection at the lateral aspect of the mesosigmoid and just as most good things in life end up in the rectum, so too does the reflection at the base of the mesosigmoid (fig. 7). The reflection at the base of the mesosigmoid converges with a similar reflection on the right side (i.e. arbitrarily called the right and left pararectal reflections) to generate the anterior peritoneal reflection in the Pouch of Douglas (fig. 7). The latter is the true termination of the peritoneal cavity in the pelvis. 


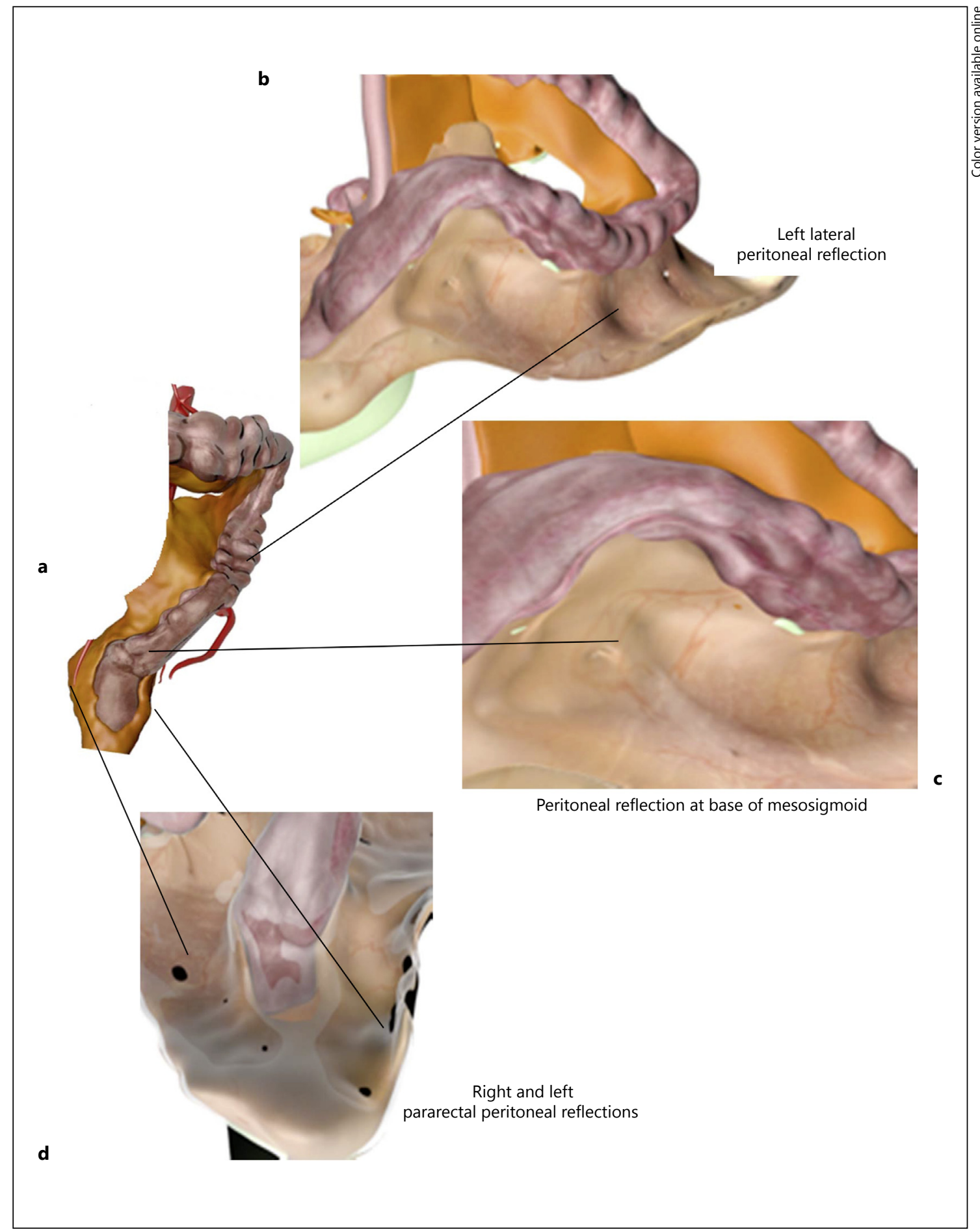

Fig. 7. a 3D digital sculpture of the colon and associated mesorectum from the splenic flexure to the rectum. b-d 3D sculptures demonstrating peritoneal reflection. $\mathbf{b}$ The model in a has been rotated to provide a lateral view of the peritoneal reflection at the lateral aspect of the descending colon. $\mathbf{c}$ The model in $\mathbf{a}$ has been rotated to show the peritoneal reflection at the base of the lateral aspect of the mesosigmoid. d View of the rectum, right and left pararectal peritoneal reflections and the region where these coalesce to form the anterior peritoneal reflection in the Pouch of Douglas. 


\section{Embryology}

The embryonic forerunner of the mesentery is the dorsal mesentery, which for a time, is contiguous from the foregut to hindgut. The gastrointestinal tract and associated mesentery concurrently undergo a complex series of elongation, rotation and then adhesion to the posterior abdominal wall. Classic embryologic descriptions were fundamentally based on the descriptions of Treves, which emphasised a fragmented and discontinuous structure. Two embryologic theories were developed as an attempt to explain the notion of right and left mesocolic regression. Given that the international community now has an alternate appraisal of mesenteric anatomy, its embryologic development must also be re-appraised. Although this is beyond the remit of the current article, any such appraisal should now be based on the anatomic principles of peritoneal, mesenteric and fascial contiguity demonstrated earlier.

\section{Summary}

The central principles of mesenteric-based surgery (including complete mesocolic excision, total mesocolic excision and total mesorectal excision) are those of intact and extensive mesenterectomy $[5,7,12,14]$. To achieve these, one requires a clear perception of peritoneal, colonic, mesenteric and fascial anatomy $[5,12,14]$. As demonstrated earlier, each of these structures is contiguous from the mesenteric origin to the termination of the mesorectum [16].

Contiguity means that anywhere from the duodenojejunal flexure distally, mobilisation can be simplified into (1) peritonotomy, (2) mesofascial and (3) colofascial separation. Adopting a stepwise approach that is fundamentally anatomic in basis, and universally reproducible (even in the context of mal or non-rotation), will help achieve the principles of CME, TME and TMCE at any level from DJ flexure to the mesorectal level.

\section{References}

1 Coffey JC: Surgical anatomy and anatomic surgery - clinical and scientific mutualism. Surgeon 2013;11:177-182.

2 Hohenberger W, Weber K, Matzel K, et al: Standardized surgery for colonic cancer: complete mesocolic excision and central ligation - technical notes and outcome. Colorectal Dis 2009;11:354-364; discussion 364-365.

3 Heald RJ: The 'Holy Plane' of rectal surgery. J R Soc Med 1988;81:503-508.

4 Heald RJ, Husband EM, Ryall RD: The mesorectum in rectal cancer surgery - the clue to pelvic recurrence? Br J Surg 1982;69:613-616.

5 Liang JT, Lai HS, Huang J, et al: Long-term oncologic results of laparoscopic D3 lymphadenectomy with complete mesocolic excision for right-sided colon cancer with clinically positive lymph nodes. Surg Endosc 2014, Epub ahead of print.

6 Karandikar S, Abbott S: Open resection for colorectal cancer. Surg (Oxford) 2014;32: 190-196.

7 Willaert W, Ceelen W: Extent of surgery in cancer of the colon: is more better? World J Gastroenterol 2015;21:132-138.
8 West NP, Morris EJ, Rotimi O, et al: Pathology grading of colon cancer surgical resection and its association with survival: a retrospective observational study. Lancet Oncol 2008; 9:857-865.

9 Bertelsen CA, Neuenschwander AU, Jansen JE, et al: Disease-free survival after complete mesocolic excision compared with conventional colon cancer surgery: a retrospective, population-based study. Lancet Oncol 2015; 16:161-168.

10 Coffey JC, Sehgal R, Culligan K, et al: Terminology and nomenclature in colonic surgery: universal application of a rule-based approach derived from updates on mesenteric anatomy. Tech Coloproctol 2014;18:789794.

11 Culligan K, Walsh S, Dunne C, et al: The mesocolon: a histological and electron microscopic characterization of the mesenteric attachment of the colon prior to and after surgical mobilization. Ann Surg 2014;260: 1048-1056.
12 Mori S, Baba K, Yanagi M, et al: Laparoscopic complete mesocolic excision with radical lymph node dissection along the surgical trunk for right colon cancer. Surg Endosc 2015;29:34-40.

13 Culligan K, Coffey JC, Kiran RP, et al: The mesocolon: a prospective observational study. Colorectal Dis 2012;14:421-428; discussion 428-430.

14 Killeen S, Kessler H: Complete mesocolic excision and central vessel ligation for right colon cancers. Tech Coloproctol 2014;18:11291131.

15 Treves F: Lectures on the anatomy of the intestinal canal and peritoneum in man. Br Med J 1885;1:580-583.

16 Kontovounisios C, Kinross J, Tan E, et al: Complete mesocolic excision in colorectal cancer: a systematic review. Colorectal Dis 2015;17:7-16.

17 Culligan K, Sehgal R, Mulligan D, et al: A detailed appraisal of mesocolic lymphangiology - an immunohistochemical and stereological analysis. J Anat 2014;225:463472. 